Check for updates

Cite this: RSC Adv., 2017, 7, 33751

Received 13th May 2017

Accepted 24th June 2017

DOI: 10.1039/c7ra05421a

rsc.li/rsc-advances

\section{Thermally shocked graphene oxide-containing biocomposite for thermal management applications $\uparrow$}

\author{
Orebotse Joseph Botlhoko, ab James Ramontja (D) a and Suprakas Sinha Ray (D) *ab \\ In this communication, we present a new approach to simultaneously improve the thermal conductivity and \\ ductility of a biobased polylactide blend composite by localizing thermally shocked graphene oxide \\ particles in a dispersed poly( $\varepsilon$-caprolactone) phase. The preliminary results presented in this work will \\ pave the way for the development of high performance environmentally benign composite materials for \\ thermal management applications.
}

\section{Introduction}

The critical issue of thermal management in electronic packaging has attracted increasing attention owing to the continuous demand for faster electronic devices and reduction in the overall size of electronic components. ${ }^{1}$ In addition, the development of environmentally benign material-based cheaper electronic devices is in the forefront of electronic industries and academic research. Ideal thermal management materials are expected to provide concurrent package protection, heat dissipation, and finally should be easily processable and environment-friendly. ${ }^{2}$ However, a combination of these properties is not easily achievable.

Conventional materials used for thermal management include metals, ceramics and carbon based fillers, and polymers like thermosets and thermoplastics. ${ }^{3,4}$ These conventional materials in their original form are not suitable for thermal management applications owing to the need for high power density and small sized electronic devices. ${ }^{5}$ For example, metals have high mass density which eliminates their use as heat sinks, and it develops stress problems during shipping and handling. Conventional polymers have low thermal conductivity and high coefficient of thermal expansion., ${ }^{3,6,7}$ One of the most promising polymers for thermal management applications is polylactide (PLA) as it is biodegradable and exhibits good mechanical properties. However, its low elongation at break, heat distortion temperature, and melt viscosity for

\footnotetext{
${ }^{a}$ Applied Chemistry Department, University of Johannesburg, Droonfontein 2028, Johannesburg, South Africa. E-mail: rsuprakas@csir.co.za; suprakas73@yahoo.com ${ }^{b}$ DST-CSIR National Centre for Nanostructured Materials, Council for Scientific and Industrial Research, Pretoria 0001, South Africa

$\dagger$ Electronic supplementary information (ESI) available: Synthesis of GO, selection of blend ratio, melt-state rheology and DSC data. See DOI: 10.1039/c7ra05421a
}

further processing are drawbacks that limit its diverse applications. ${ }^{8}$

To overcome the above-mentioned drawbacks, several studies on graphene particles-filled PLA/PCL blend composites have been conducted. For example, Huang et al. ${ }^{9}$ showed the improved efficiency of selectively located graphene fillers at the interface of immiscible PCL/PLA blends; the PCL and the PLA phases formed a co-continuous structure resulting in high thermal conductivity. Mosanenzadeh et al. ${ }^{2}$ reported that a higher filler loading of combined graphene nanoplatelets/ hexagonal boron nitride in PLA composites resulted in higher thermal conductivity than a lower loading in graphene nanoplatelets/hexagonal boron nitride-filled PLA composites. On the other hand, Huang et al. ${ }^{10}$ demonstrated improved thermal conductivity with a high filler loading of combined carbon nanotubes/graphene nanoplatelets in epoxy matrix. Several studies revealed that thermal conductivity of graphenebased composites has the potential to outperform thermal conductivity of composites filled with carbon nanotubes or metal particles due to unique two-dimensional phonon transport, physical properties, and very high thermal conductivity of graphene. This indicates that the inherent properties of the filler are vital for obtained composite properties. ${ }^{11-14}$ In addition, few studies demonstrating the enhanced thermal conductivity of boron nitride-filled composites at a high filler loading have been reported..$^{15,16}$

In brief, very high filler loading and trapping of the filler at the interface of immiscible polymer blends strongly affects the transport phenomena; hence, blend composites exhibit high thermal conductivity. ${ }^{9,16}$ Furthermore, surface treatment of the filler using strong acids or acetone helps in controlling the filler localization in the system, which results in high thermal conductivity. Mixing particles of different sizes and shapes has also been demonstrated as an effective method to improve the thermal conductivity. ${ }^{16,17}$ However, these fundamentals are not in line with the interests of the $21^{\text {st }}$ century technology of 
electronic industries. Considering the filler loading, localization, dispersibility, and the above-mentioned drawbacks of PLA, it is clear that there is a need for research and development of environmentally benign thermal management components.

The aim of this communication is to demonstrate the potential of graphene oxide (GO)-based filler for thermal management applications, since graphene has very high thermal conductivity of approximately $5.5 \times 10^{3} \mathrm{~W} \mathrm{~m}^{-1} \mathrm{~K}^{-1}$, theoretical specific surface area of approximately $2630 \mathrm{~g} \mathrm{~m}^{-2}$, and other unique physical characteristics. ${ }^{18,19}$ In addition, we present an interesting example of highly dispersible and selfcontrollable GO-based material obtained through one-step green chemistry based protocol. The concurrent improvements in the thermal conductivity and the electrical resistivity with the addition of only GO-based filler in the PLA/PCL blend and the dependence of the compatibilization efficiency on the filler dispersibility have been studied.

\section{Experimental}

The graphite (G) powder used to prepare GO in this study was purchased from Sigma-Aldrich, South Africa. A commercialgrade (PLA U'Z S-17) PLA (D-content $\approx 1 \%$ ) obtained from Toyota, Japan with $M_{\mathrm{w}}=120-135 \mathrm{~kg} \mathrm{~mol}{ }^{-1}, \rho=1.24 \mathrm{~g} \mathrm{~cm}^{-3}$, a melt flow index (MFI) of $15.84 \mathrm{~g} / 10 \mathrm{~min}\left(190{ }^{\circ} \mathrm{C} / 2.16 \mathrm{~kg}\right.$, according to the ISO 1133B standard method), $T_{\mathrm{g}}=62{ }^{\circ} \mathrm{C}$, and $T_{\mathrm{m}}=175{ }^{\circ} \mathrm{C}$ was used in this study. PCL was purchased from Sigma-Aldrich, South Africa. According to the supplier, the properties of PCL were $M_{\mathrm{w}}=80 \mathrm{~kg} \mathrm{~mol}^{-1}, \rho=1.145 \mathrm{~g} \mathrm{~cm}^{-3}$, MFI $=5.57 \mathrm{~g} / 10 \mathrm{~min}\left(190{ }^{\circ} \mathrm{C} / 2.16 \mathrm{~kg}\right.$, according to the ISO $1133 \mathrm{~B}$ standard method), and $T_{\mathrm{m}}=60{ }^{\circ} \mathrm{C}$. Prior to use, PLA was dried at $80{ }^{\circ} \mathrm{C}$, while PCL was dried at $40{ }^{\circ} \mathrm{C}$ under vacuum for $12 \mathrm{~h}$.

GO was prepared through chemical oxidation of the natural G powder according to the well-known improved Hummers' method reported by the Tour group, ${ }^{20}$ as presented briefly in the ESI. $\uparrow$ To obtain thermally exfoliated and partially reduced GO (TERGO), the dried GO powder $(\sim 0.1 \mathrm{~g})$ was subjected to thermal shock in a tube furnace at $700{ }^{\circ} \mathrm{C}$ for a minute under the flow of argon gas $\left(120 \mathrm{ml} \mathrm{min}^{-1}\right)$.

Neat blend and blend composites were prepared via meltmixing in a HAAKE PolyLab OS Rheomix (Thermo Electron Co., USA) operated at a rotor speed of $60 \mathrm{rpm}$ at a temperature of $195{ }^{\circ} \mathrm{C}$ for $8 \mathrm{~min}$. All the components were dry-mixed in a plastic bag before melt mixing. The neat PLA/PCL blend and the blend composites were then compression molded into different shapes at $195{ }^{\circ} \mathrm{C}$ using a Carver $973214 \mathrm{~A}$ hot press at $1.2 \mathrm{MPa}$ and cooled to $\pm 20{ }^{\circ} \mathrm{C}$. However, the samples for thermal conductivity measurements were melt-pressed at $195{ }^{\circ} \mathrm{C}$ under a pressure of $50 \mathrm{kPa}$ using a custom-built 20-ton hydraulic melt press. The samples containing 0 and $0.05 \mathrm{wt} \%$ of either GO or TERGO filler are denoted as blend, blend/GO, and blend/ TERGO, respectively. Neat PLA and PCL were prepared by the same method as the neat blend and its composites. Furthermore, the PLA/PCL blend weight ratio was fixed at 60:40 and finally denoted as blend. The motivation to select this blend ratio can be found in the ESI (Fig. S1, ESI). $\dagger$
The chemical and structural characterizations of the G, GO, and TERGO particles were carried out using attenuated total reflectance Fourier-transform infrared spectra (FTIR) spectroscopy with a Perkin-Elmer Spectrum 100 spectrometer (USA) in the range of 600 to $4000 \mathrm{~cm}^{-1}$. The morphological analyses of the GO and the TERGO particles were carried out by fieldemission scanning electron microscopy (FESEM; JSM-7500, JEOL, Japan). The samples were sputter coated with carbon before imaging at an acceleration voltage of $2 \mathrm{kV}$. Raman spectroscopic measurements were conducted using a JobinYvon T64000 Raman spectrometer (France) equipped with an OlympusBX40 micro-Raman (100× objective) using $514.5 \mathrm{~nm}$ excitation wavelength of an argon ion-laser. The morphologies of the polymeric samples were also analyzed using FESEM. The blend and the composite samples were first freeze-fractured in liquid nitrogen, and then the PCL minor phase was etched with acetic acid for $15 \mathrm{~h}$ at $27{ }^{\circ} \mathrm{C}$. The etched surface was then sputter-coated with carbon, and the sides were painted with a silver paste and finally imaged at an acceleration voltage of 3 $\mathrm{kV}$. The number average radius $\left(R_{\mathrm{n}}\right)$ of the etched and dispersed PCL droplets were determined by imageJ software (ImageJ 1.46r), using eqn (1).

$$
R_{\mathrm{n}}=\frac{\sum n_{i} R_{i}}{n_{i}}
$$

where, $n_{i}$ is the number of dispersed droplets with a radius $R_{i}$. Furthermore, the morphological characteristics of the GO, TERGO particles, GO- and the TERGO-filled blend composites were studied by high-resolution transmission electron microscopy (HRTEM; JEM2100, JEOL, Japan) at an acceleration voltage of $200 \mathrm{kV}$. Tensile tests of dog-bone-shaped neat PLA, blend, and composite samples were carried out with an Instron 5966 tester (Instron Engineering Corp., USA) using a load cell of 10 $\mathrm{kN}$ at a tension mode rate of $5 \mathrm{~mm} \min ^{-1}$ at approximately $30{ }^{\circ} \mathrm{C}$. The dog-bone-shaped samples were approximately $3 \mathrm{~mm}$ thick and $3.40 \mathrm{~mm}$ wide, and the gauge length was $25 \mathrm{~mm}$. The reported results are an average of at least six independent tensile test measurements. The thermal conductivity measurements were performed at approximately $24^{\circ} \mathrm{C}$ on discs with 6 $\mathrm{mm}$ thickness and $13 \mathrm{~mm}$ diameter using a ThermoTest Inc (Hot Disk TPS 500, Sweden) thermal conductivity analyzer (TCA). The instrument uses the transient plane source method. A $3.2 \mathrm{~mm}$ Kapton disk type sensor was selected for the analysis with a power of approximately $2.1 \mathrm{~W}$. The sensor was sandwiched between two disc-shaped samples. Three measurements were performed for each composition. The electrical resistance measurements were performed at $27{ }^{\circ} \mathrm{C}$ using a four point probe station (Cascade Microtech, C4S 54 28833, USA) connected to a Keithley 4200 Semiconductor Characterization System. Disc-shaped samples of approximately $25 \mathrm{~mm}$ diameter and $1.68 \mathrm{~mm}$ thickness were used. The electrical resistivity ( $\rho$, ohm per $\mathrm{cm}$ ) was estimated according to eqn (3):

$$
\rho=R \frac{A}{L}
$$




$$
\rho=\frac{\pi r^{2} R}{L}
$$

where, $R$ is the electrical resistance (ohms) obtained from the instrument, $L$ is the length considered as the thickness of the sample $(0.164 \mathrm{~mm})$, and $A$ is the cross-sectional area $\left(A=\pi r^{2}\right)$ of the sample calculated using radius $r$ (diameter $\times 1 / 2$ in $\mathrm{mm}$ ). Thermal characteristics of the prepared polymeric samples weighing approximately $5.6 \mathrm{mg}$ were determined by differential scanning calorimetry (DSC) (model Q2000, TA Instruments., USA) from -65 to $200^{\circ} \mathrm{C}$ at a scanning rate of $10^{\circ} \mathrm{C} \mathrm{min}^{-1}$ under

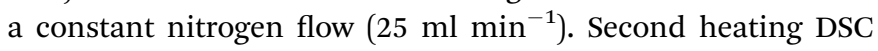
scans are presented, since first scan was used to erase the thermal history effects. Degree of crystallinity $\left(X_{\mathrm{c}}\right)$ was estimated according to eqn (4).

$$
\% \text { crystallinity }\left(\% \chi_{\mathrm{c}}\right)=\frac{\Delta H_{\mathrm{m}}}{\Delta H_{\mathrm{m}}^{\circ} \times W_{\mathrm{f}}} \times 100
$$

where $\Delta H_{\mathrm{m}}$ is the specific melting enthalpy of each polymer phase in a sample, $W_{\mathrm{f}}$ is the weight fraction of each polymer within the sample, and $\Delta H_{\mathrm{m}}^{\circ}$ is the specific melting enthalpy of $100 \%$ crystalline PLA or PCL, which are 93 (ref. 21) or 135 (ref. 22) $\left(\mathrm{J} \mathrm{g}^{-1}\right)$, respectively.

\section{Results and discussion}

The FTIR spectra and the SEM images of the GO and the TERGO particles are presented in Fig. 1(a), (b) and (c) respectively. No
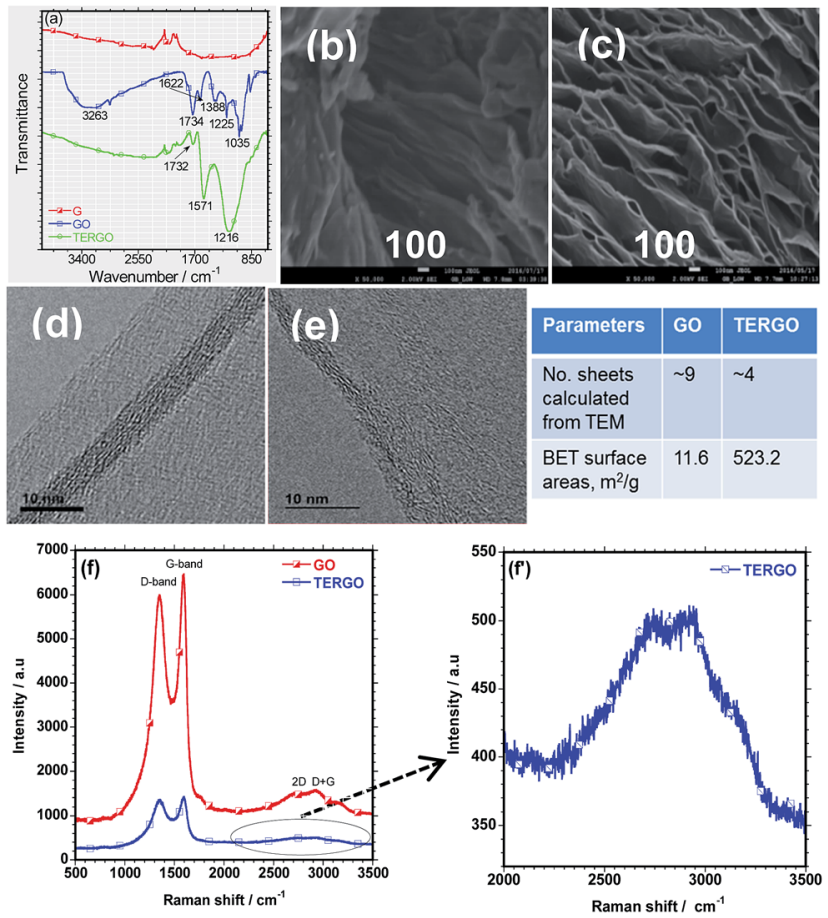

Fig. 1 (a) The FTIR spectra of the GO and the TERGO particles, (b) the SEM of the GO particles, (c) the SEM image of TERGO particles, (d) the TEM image of the GO particles, and (e) the TEM image of TERGO particles. The characteristic parameters of GO and TERGO are presented in inset table. (f and $f^{\prime}$ ) Raman spectra of GO and TERGO. well-defined IR peaks were observed in the FTIR spectrum of G. On the other hand, the spectrum of GO was characterized by IR peaks at 1035, 1225, 1388, 1622, 1734, and $3263 \mathrm{~cm}^{-1}$, which correspond to the alkoxy $\mathrm{C}-\mathrm{O}$, epoxy $\mathrm{C}-\mathrm{O},-\mathrm{OH}$ bending deformation (bound $\mathrm{H}_{2} \mathrm{O}$ molecules) or $\mathrm{C}-\mathrm{O}$ vibration, $\mathrm{C}=\mathrm{C}$ stretching of the aromatic ( $\mathrm{sp}^{2}$ system of $\left.\mathrm{GO}\right), \mathrm{C}=\mathrm{O}$ stretching vibration of the carboxyl group, and hydroxyl - $\mathrm{OH}$ (free $\mathrm{H}_{2} \mathrm{O}$ molecules), respectively. ${ }^{23-25}$ The presence of these IR peaks confirm the successful oxidation of GO. The FTIR spectrum of TERGO was characterized by similar IR peaks at 1216, 1571, and $1732 \mathrm{~cm}^{-1}$, corresponding to the stretching vibration of the C$\mathrm{O}, \mathrm{C}=\mathrm{C}$, and $\mathrm{C}=\mathrm{O}$ bonds, respectively that exist in both the GObased particles. It is evident that the thermal exfoliation process of GO removed most of the oxygen-containing functional groups and decreased the intensity of the remaining IR peaks.

The SEM image of GO showed the presence of thin graphene sheets and a wrinkled surface morphology [Fig. 1(b)]. On the other hand, the SEM image of TERGO showed well-expanded and thinner graphene sheets compared to those of GO. The decrease in the thickness indicates the removal of bound water and other oxygen-containing functional groups as discussed previously, indicating successful exfoliation. The expansion suggests an increase in the volume ratio. To support the above findings and obtain more insights into the oxidation of GO, exfoliation and slight reduction of the TERGO, Raman spectroscopic analyses were conducted. Fig. 1(f) shows the Raman spectra of the GO and the TERGO particles. The Raman spectra revealed that GO contains narrow D- and G-band peaks at about $1349.83 \mathrm{~cm}^{-1}$ and $1595.92 \mathrm{~cm}^{-1}$ due to the presence of hydroxyl, epoxy, and carboxyl functional groups. ${ }^{20,26}$ In addition, very weak $2 \mathrm{D}$ and $\mathrm{D}+\mathrm{G}$ peaks are also visible at $2727.43 \mathrm{~cm}^{-1}$ and $2931.42 \mathrm{~cm}^{-1}$, indicating multi-layer graphene sheets. ${ }^{26} \mathrm{On}$ the other hand, TERGO contains characteristic D- and G-band peaks at $1355.47 \mathrm{~cm}^{-1}$ and $1591.79 \mathrm{~cm}^{-1}$. Significantly weak $\mathrm{D}$ and $\mathrm{D}+\mathrm{G}$ peaks at about $2743.49 \mathrm{~cm}^{-1}$ and $2921.44 \mathrm{~cm}^{-1}$ are observed in the case of TERGO (Fig. 1(f')). Importantly, the intensity of the D and G peaks and their overtones significantly decreased upon thermal shocking process. Thus, supports the expansion and reduction of number graphene sheets due to the removal of most of oxygen-containing functional groups [Fig. 1(a)-(e)] and inset table, which lead to high thermal conductivity characteristics. The data obtained based on the Dand G-peaks, and their overtones are consistent with chemical oxidation, thermal exfoliation and reduction reports. ${ }^{20,26,27}$

The freeze-fractured PCL-etched surface morphology of the neat blend presented in Fig. 2(a) shows that the irregular PCL droplets are non-uniformly dispersed in the PLA matrix, suggesting poor compatibility between the PLA and the PCL phases. The droplet size $\left(R_{\mathrm{n}}, \mu \mathrm{m}\right)$ of the dispersed PCL minor phase decreased when the GO [Fig. 2(b)] and the TERGO [Fig. 2(c)] particles were incorporated in the blend. For example, in the case of the neat blend, the calculated size of the dispersed phase was $1.31 \pm 0.26 \mu \mathrm{m}$, which decreased to $0.79 \pm 0.25 \mu \mathrm{m}$ and 0.76 $\pm 0.04 \mu \mathrm{m}$ in the cases of GO- and TERGO-filled blend composites, respectively. It is notable that the addition of the TERGO particles improved the uniformity of the dispersed PCL droplets. Such an observation is attributed to the preferential 


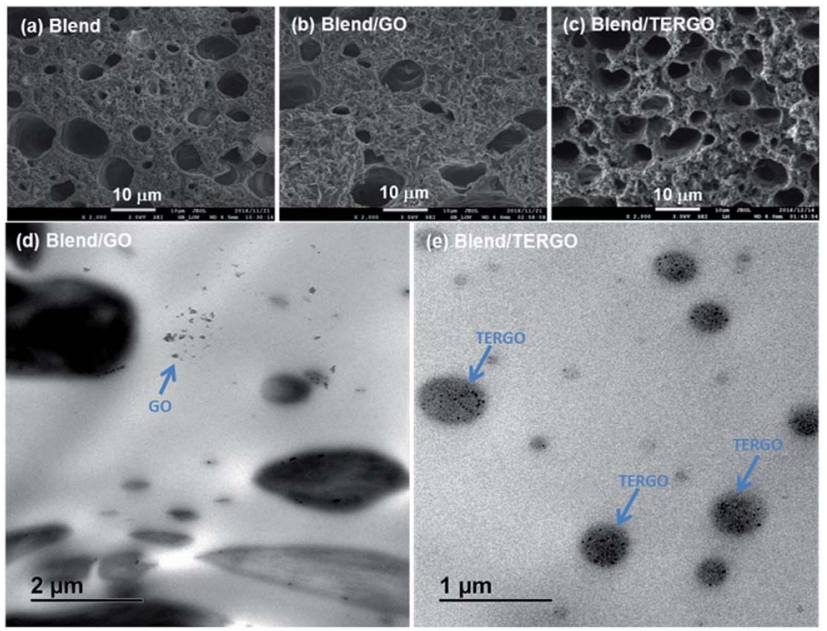

Fig. 2 The freeze-fractured PCL-etched surface morphology of the (a) neat blend, (b) GO-filled blend composite, and (c) TERGO-filled blend composite. TEM images of (d) GO-filled blend composite and (e) TERGO-filled blend composite. The arrows show the position of GO and TERGO particles in blend matrix.

localization of the TERGO particles in the dispersed PCL phase, resulting in an increased viscosity ratio, which in turn leads to a delay of or an insufficient coalescence of the dispersed PCL droplets. This observation indicates that $0.05 \mathrm{wt} \%$ TERGO is sufficient to fine-tune and stabilize the phase-separated blend morphology. The stable and compatibilized morphology of the TERGO-filled blend composite conduced to a higher melt viscosity (Fig. S2, ESI†).

To support the above findings and obtain more insights on the dispersions of the GO and the TERGO particles in the PLA/ PCL phase, local TEM analyses were carried out. Fig. 2(d) and (e), respectively, show the bright field TEM images of the GOand the TERGO-filled blend composites. The TEM micrographs revealed that most of the GO particles were randomly dispersed in the PLA phase and very few particles existed in the PCL phase. On the contrary, the TERGO particles were homogeneously dispersed in the PCL phase.

From Fig. 3, it is noticeable that the neat PLA exhibits high modulus and tensile strength and a very low elongation at break. The observed brittleness of PLA is a major disadvantage for various applications. ${ }^{21}$ Upon blending PLA with PCL, the modulus and the tensile strength decreased significantly, however, the ductile nature of PCL led to a significant increase in the elongation at break of the PLA/PCL blend. On the other hand, the GO-filled blend composite showed deterioration of all the tensile properties, which is attributed to the stiffness of the GO particles and the poor compatibilization efficiency. Interestingly, the TERGO-filled blend composite showed a balance of modulus and tensile strength compared to those of the neat blend and a significant increase in the elongation at break. This indicates that the TERGO particles are excellent compatibilization agents for this blend. It should be noted that the error bars of the TERGO-filled blend composite were significantly smaller than those of the GO-filled blend composite, indicating
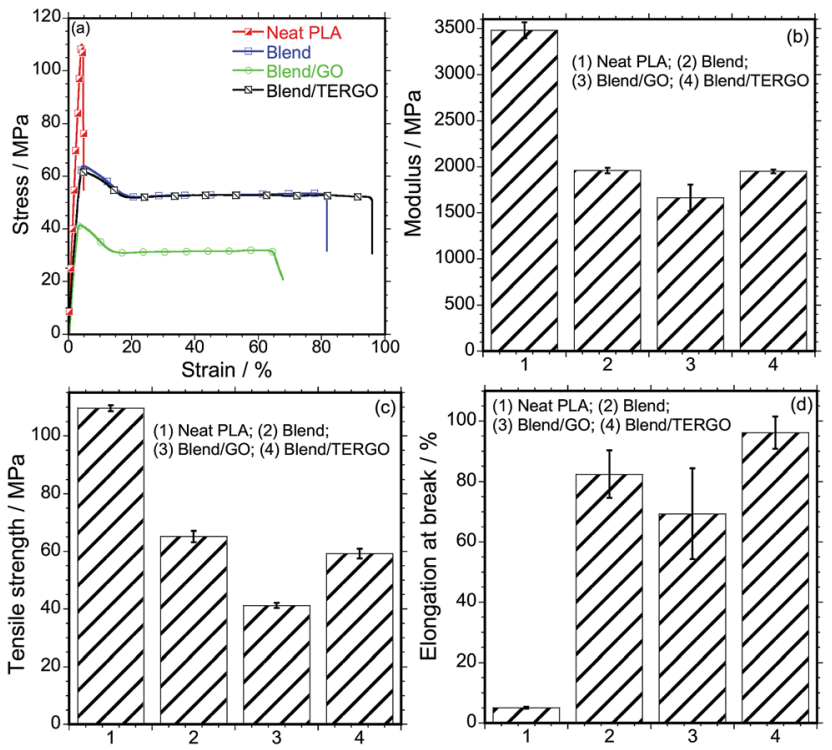

Fig. 3 (a) Stress-strain curves, (b) tensile modulus, (b) tensile strength, and (d) elongation-at-break of neat polymers, blend, and GO- and TERGO-filled blend composites.

better dispersion of the PCL droplets in the PLA matrix due to the preferential localization of the TERGO particles.

It is known that heat transport in G particles or its derivatives occurs via phonons of varying frequencies. The high frequency phonons are the major heat energy carriers, and the low frequency phonon vibrations carry a small amount of heat energy in various systems. ${ }^{28}$ In order to elucidate the thermal conductivity and the electrical resistivity behaviors of neat PLA, PCL, blend, GO- or TERGO-filled blend composites, the transport properties were measured, and the relative thermal conductivity and resistivity bar graphs are shown in Fig. 4(a) and (b), respectively. It is notable that the neat PLA has a higher thermal conductivity $\left(0.22392 \mathrm{~W} \mathrm{~m}^{-1} \mathrm{~K}^{-1}\right)$ and resistivity $\left(1.8959 \times 10^{13} \mathrm{ohm}\right.$ per $\left.\mathrm{cm}\right)$ than the neat PCL $\left(0.17312 \mathrm{~W} \mathrm{~m}^{-1}\right.$ $\mathrm{K}^{-1}$ and $1.2716 \times 10^{13} \mathrm{ohm}$ per $\left.\mathrm{cm}\right)$ and the blend $(0.20821 \mathrm{~W}$ $\mathrm{m}^{-1} \mathrm{~K}^{-1}$ and $8.4007 \times 10^{12} \mathrm{ohm}$ per $\mathrm{cm}$ ). The obtained value of the thermal conductivity of neat PLA is consistent with its known value of approximately $0.16 \mathrm{~W} \mathrm{~m}^{-1} \mathrm{~K}^{-1}$. ${ }^{2}$ Upon blending PLA with PCL, a slight reduction in the thermal conductivity with a significant reduction in the resistivity was observed. The slight reduction in the thermal conductivity of the blend is attributed to the low thermal conductivity of the dispersed PCL droplets. The resistivity behavior of the blend is unusual since polymers are natural insulators, and thus, an enhanced electrons blockage is expected. Therefore, the significant reduction in the resistivity is attributed to the effects of fairly pinched spherulites, mixture of large and small spherulites (Fig. 5), or non-uniform dispersed PCL droplets [Fig. 2(a)] which act as electrically conducting pathways.

It is evident that the TERGO-filled blend composite exhibited the highest thermal conductivity value, which is significantly higher than those of the neat blend and the GO-filled blend composite as well as those of the neat PLA and PCL, due to high 

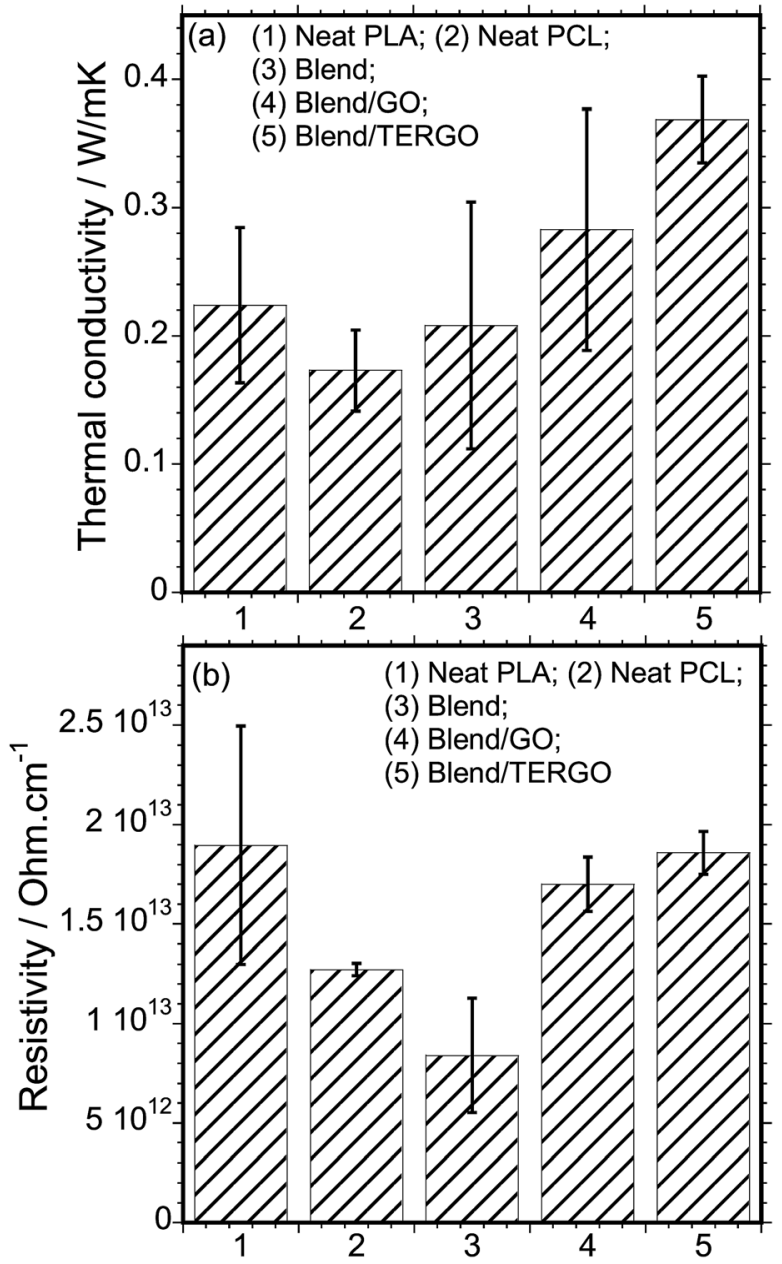

Fig. 4 (a) The thermal conductivity and (b) the electrical resistivity behaviors of neat PLA, PCL, blend, GO- or TERGO-filled blend composites. The filler loading was fixed at $0.05 \mathrm{wt} \%$.

frequency phonon and few layers of graphene sheets as results of high temperature treatment. ${ }^{29,30}$ Again, the error bars of the TERGO-filled blend composite were significantly smaller than the neat blend and the GO-filled blend composite. This confirms that the PCL droplets are uniformly dispersed in the PLA matrix [Fig. 2(c)] because of the strong affinity of the TERGO particles towards the PCL phase and the selfcontrollable homogeneous dispersion of the TERGO particles [Fig. 2(e)]. The attained uniform surface morphology and the predominance of TERGO particles in the PCL minor phase give rise to particle-particle interactions that lead to more phonon scattering interfaces and short distance phonon transportation within the blend, increasing the thermal conductivity and the resistivity simultaneously.

However, the lower thermal conductivity of the GO-filled blend composite relative to the TERGO-filled composite is attributed to the insufficient phonon scattering interfaces at the boundaries and longer distances for GO particle-particle interactions in the system, which leads to smaller thermal contact areas. The unique electrical resistivity for GO-filled blend composite is attributed to the introduction of oxide

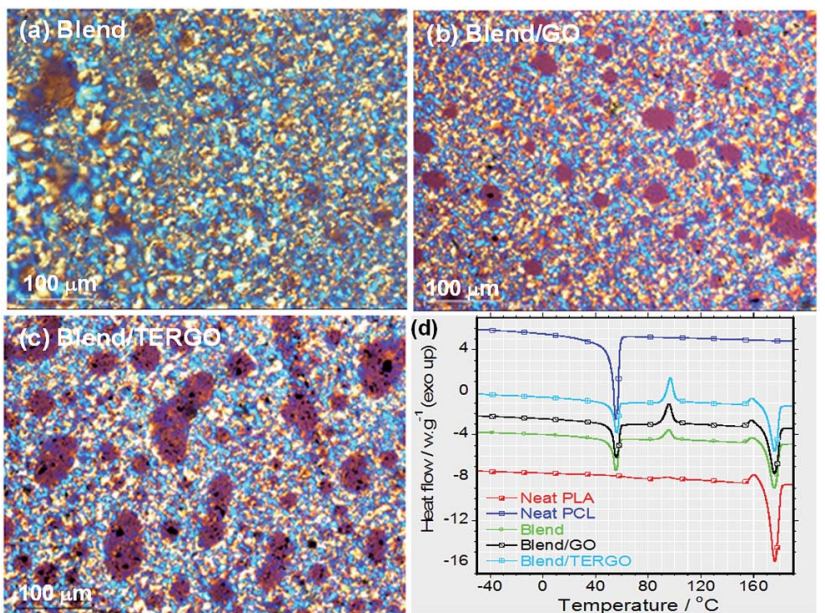

Fig. 5 Polarized optical microscopic images of isothermal (at $120^{\circ} \mathrm{C}$ ) crystallized samples of (a) neat blend, (b) GO-filled blend composite, and (c) TERGO-filled blend composite. (d) Differential scanning calorimetric thermograms of various samples during heating after crystallization from their melting states. Thin film samples were heated to $200{ }^{\circ} \mathrm{C}$, cooled to $120^{\circ} \mathrm{C}$ at a rate of $10^{\circ} \mathrm{C} \mathrm{min}^{-1}$, isothermal at this temperature for $20 \mathrm{~min}$ and spherulitic growth behavior was observed using Carl Zeiss Imager Z1M polarized optical microscope. Prior to POM analysis DSC measurements were conducted.

groups, such as epoxy, hydroxyl or carboxyl [Fig. 1(a)]. They play the key role by trapping electrons, hence electrically insulating behaviour is notable. ${ }^{31}$ While the presences of few oxygenated sites on the TERGO particles act in the same way as those of GO particles, however the enhanced electrical resistivity of TERGOfilled blend composite relative to GO-filled blend composite is due to perfectly located TERGO particles in the PCL minor phase as well as poor electrical network and the use of PLA matrix pathway for electrical transport. Based on the obtained results, it is proposed that the thermal conductivity and the electrical resistivity characteristics are strongly influenced by the surface morphology, particle-particle filler interaction, volume ratio, and phonon scattering within the polymeric system.

To understand the thermal behavior of neat polymers, neat blend, and blend composite, DSC analyses were conducted and the second heating scans are shown in Fig. 5(d), while the characteristic parameters calculated from DSC thermograms are summarized in Table S1. $\dagger$ Neat PLA is characterized by high $T_{\mathrm{m}}$ value of $176.91{ }^{\circ} \mathrm{C}$ and $X_{\mathrm{c}}$ of approximately $59.42 \%$, while neat PCL is characterized by low $T_{\mathrm{m}}$ of $55.48{ }^{\circ} \mathrm{C}$ and $X_{\mathrm{c}}$ of approximately $46.39 \%$. The obtained melting profiles for neat PLA and PCL are in agreement with the published melting profiles of these individual polymers, $\left(\mathrm{PLA}^{32,33}\right.$ and $\mathrm{PCL}^{34}$ ). In case of the blend, both PLA and PCL melting profiles are observed within the same range respective to their neat polymers, however the $X_{\mathrm{c}}$ of PLA slightly decreased to $58.30 \%$ while the one relative to PCL significantly decreased to $31.91 \%$. The achieved melting profiles indicate immiscibility behavior of the blend, which is corroborating with SEM images (Fig. 2(a)). Upon the addition of GO and TERGO particles, the melting profiles of the composites shows similar melting values as that of neat 
blend (Table S1 $\dagger$ ). However, the presence of GO particles improved the $X_{\mathrm{c}}$, while TERGO particles reduced the $X_{\mathrm{c}}$ relative to that of the neat blend. The change in $X_{\mathrm{c}}$ between the blend and its GO- or TERGO-filled composites suggests the alteration of spherulites size and arrangements (Fig. 5(a)-(c)) as well as the mobility of the polymer chains,$^{35}$ which is justified by variation in melt viscosity (Fig. S2, ESI $\dagger$ ).

\section{Conclusions}

In summary, the dispersed TERGO particles demonstrated the potential to fine-tune and stabilize the surface morphology of the PLA/PCL blend without significantly altering the tensile strength and the modulus. Such a stable morphology resulted in a notable improvement in the elongation at break of the TERGO-filled blend composite. The thermal conductivity and the electrical resistivity of the GO- and the TERGO-filled blend composites were increased relative to the neat blend. However, the TERGO-filled blend composite exhibited optimum values for both characteristics. Thus, lightweight, tough, and fairly flexible TERGO-filled blend composites have the potential for thermal management and other packaging applications, however, intensive studies still need to be conducted.

\section{Acknowledgements}

The authors appreciate the financial support of the National Research Foundation, South Africa together with the University of Johannesburg and the Council for Scientific and Industrial Research, and further thank the staff of the DST-CSIR NCNSM characterization facility for their work on characterization.

\section{Notes and references}

1 R. Solomon, P. Sandborn and M. Pecht, IEEE Trans. Compon. Packag. Technol., 2000, 707-717.

2 S. G. Mosanenzadeh, S. Khalid, Y. Cui and H. E. Naguib, Polym. Compos., 2016, 37, 2196-2205.

3 H. Ding, Y. Guo and S. N. Leung, J. Appl. Polym. Sci., 2016, 133, DOI: 10.1002/app.42910.

4 M. T. Sebastian, Int. J. Appl. Ceram. Technol., 2010, 7, 415434.

5 G. Subodh, C. Pavithran, P. Mohanan and M. T. Sebastian, J. Eur. Ceram. Soc., 2007, 27, 3039-3044.

6 J. Gu, X. Yang, Z. Lv, N. Li, C. Liang and Q. Zhang, Int. J. Heat Mass Transfer, 2016, 92, 15-22.

7 R. K. Goyal, P. Jadhav and A. N. Tiwari, J. Electron. Mater., 2011, 40, 1377-1383.

8 S. S. Ray, K. Yamada, A. Ogami, M. Okamoto and K. Ueda, Macromol. Mater. Eng., 2002, 23, 943-947.

9 J. Huang, Y. Zhu, L. Xu, J. Chen, W. Jiang and X. Nie, Compos. Sci. Technol., 2016, 129, 160-165.

10 X. Huang, C. Zhi and P. Jiang, J. Phys. Chem. C, 2012, 116, 23812-23820.
11 K. M. F. Shahil and A. A. Balandin, Nano Lett., 2012, 12, 861867.

12 J. D. Renteria, D. L. Nika and A. A. Balandin, Appl. Sci., 2014, 4, 525-547.

13 D. L. Nika and A. A. Balandin, J. Phys.: Condens. Matter, 2012, 24, 233203.

14 A. A. Balandin, Nat. Mater., 2011, 10, 569-581.

15 H. Ishida and S. Rimdusit, Thermochim. Acta, 1998, 320, 177186.

16 M. Donnay, S. Tzavalas and E. Logakis, Compos. Sci. Technol., 2015, 110, 152-158.

17 V. Goyal and A. A. Balandin, Appl. Phys. Lett., 2012, 100, 073113.

18 J. Wang, F. Ma and M. Sun, RSC Adv., 2017, 7, 16801-16822. 19 F. You, X. Li, L. Zhang, D. Wang, C. Shi and Z. Dang, RSC Adv., 2017, 7, 6170-6178.

20 D. C. Marcano, D. V. Kosynkin, J. M. Berlin, A. Sinitskii, Z. Sun, A. Slesarev, L. B. Alemany, W. Lu and J. M. Tour, ACS Nano, 2010, 4, 4806-4814.

21 J. Ramontja, S. S. Ray, S. K. Pillai and A. S. Luyt, Macromol. Mater. Eng., 2009, 294, 839-846.

22 K. K. Gupta, N. Pal, P. K. Mishra, P. Srivastava, S. Mohanty and P. Maiti, J. Biomed. Mater. Res., Part A, 2014, 102, 2600-2612.

23 B. T. K. Sharmila, A. B. Nair, B. T. Abraham, P. M. S. Beegum and E. T. Thachil, Polymer, 2014, 55, 3614-3627.

24 Y. Yan, T. Kuila, N. H. Kim and J. H. Lee, Carbon, 2014, 74, 195-206.

25 W. Chen, L. Yan and P. R. Bangal, Carbon, 2010, 48, 11461152.

26 A. C. Ferrari, J. C. Meyer, V. Scardaci, C. Casiraghi, M. Lazzeri, F. Mauri, S. Piscanec, D. Jiang, K. S. Novoselov, S. Roth and A. K. Geim, Phys. Rev. Lett., 2006, 97, 187401.

27 Y. Yan, T. Kuila, N. H. Kim and J. H. Lee, Carbon, 2014, 74, 195-206.

28 M. J. Mochane and A. S. Luyt, Polym. Eng. Sci., 2015, 55, 1255-1262.

29 D. L. Nika and A. A. Balandin, Rep. Prog. Phys., 2017, 80, 036502.

30 J. D. Renteria, S. Ramirez, M. Hoda, B. Alonso, A. Centeno, A. Zurutuza, A. I. Cocemasov, D. L. Nika and A. A. Balandin, Adv. Funct. Mater., 2015, 25, 4664-4672.

31 P. K. S. Mural, M. Sharma, G. Madras and S. Bose, RSC Adv., 2015, 5, 32078-32087.

32 A. K. Matta, R. U. Rao, K. N. S. Suman and V. Rambabu, Procedia Mater. Sci., 2014, 6, 1266-1270.

33 V. Ojijo, S. S. Ray and R. Sadiku, ACS Appl. Mater. Interfaces, 2012, 4, 2395-2405.

34 S. C. Agwuncha, S. S. Ray, J. Jayaramudu, C. Khoathane and R. Sadiku, Macromol. Mater. Eng., 2015, 300, 31-47.

35 M. J. Hato, S. S. Ray and A. S. Luyt, Macromol. Mater. Eng., 2008, 293, 752-762. 